\title{
An Integrated Methodology for Approaching Sentiment Analysis in Business Domain
}

\author{
Fernando Ferri ${ }^{1}$, Alessia D’Andrea ${ }^{1}$, Patrizia Grifoni ${ }^{1}$ \\ ${ }^{1}$ National Research Council, Italy \\ Correspondence: Alessia D‘Andrea, National Research Council, Italy.
}

Received: June 23, 2017

Accepted: July 27, $2017 \quad$ Online Published: August 1, 2017

doi:10.5539/ibr.v10n9p1

URL: https://doi.org/10.5539/ibr.v10n9p1

\begin{abstract}
The paper provides a methodology for approaching sentiment analysis in the business domain. It involves the different phases that compose the sentiment analysis processing (sentiment extraction, sentiment changes detection and sentiment prediction). Each phase involves different steps and some examples of approaches/methods/models to perform them. An example of an application scenario for the methodology is defined in the paper.
\end{abstract}

Keywords: sentiment analysis, social media, sentiment extraction, sentiments chances detection; sentiment prediction

\section{Introduction}

With the widespread diffusion of Social Media applications such as Social Networks, forums, micro-blogging etc., a lot of comments, opinions, and feedbacks generated by consumers are available. With the explosion of consumer's generated contents, comes the need by companies to analyse them in order to evaluate and improve the efficiency of their business strategies. In this context, the sentiment analysis plays an important role. Sentiment analysis "has arisen in a context that is said to be characterized by an overabundance of corporate, marketing messages, a decline of trust in advertisements, and a growth of trust in peer recommendations" (Kennedy, 2012). In D'Andrea et al. (2012) the authors explain that sentiment analysis aims "to identify how sentiments are expressed in texts and whether the expressions indicate positive (favorable) or negative (unfavorable) opinions toward the subject". In the business domain, the sentiment analysis is used for analysing customers' feelings towards a particular product/service to understand how consumers respond and improve the business strategies. Companies often use sentiment analysis for discovering unique strategies for their advertising campaigns, whether it's for online banner ads or direct mail postcards. The overall purpose is to build loyalty with consumers, who help companies to grow and expand. Due to the widespread diffusion of sentiment analysis, different studies have been provided in the literature with the aim to develop approaches for sentiment analysis processing in business domain (Fan et al., 2011; Wang et al., 2013). However the existing literature in this field appears fragmented: in some studies only a description of the sentiment extraction phase is provided (Rastogi et al., 2014; Duwairi et al., 2014; Khan et al., 2014; Bagwan et al., 2013; De Clercq et al., 2014) while other studies only consider sentiment changes detection (Bifet at al., 2011; Daas \& Puts, 2014; Nguyen et al., 2012) and/or sentiment prediction (Park, 2004; Madrigal, 2001; Zhang et al., 2011a; Bollen et al., 2011; Gilbert \& Karahalios, 2010). In order to overcome this fragmentation, in this paper an integrated methodology for approaching sentiment analysis in business domain is provided. The methodology is composed of the different phases that characterize the sentiment analysis process (sentiment extraction, sentiment changes detection and sentiment prediction). Each phase involves different steps and some examples of approaches/methods/models to perform them. An example of the use of the methodology for extracting user's sentiment on financial services is provided in the paper. The paper is structured as follow. Section 2 provides a literature review on different phases provided in the literature for analysing users' sentiments on Social Media. In Section 3 the methodology is provided. In Section 4 an example of an application scenario for the methodology is described. In Section 5 a discussion on the contribution of the methodology is provided. Finally, Section 6 concludes the paper.

\section{Related Work}

Business represents an important activity for any company, as it explains how the company will earn revenue (Ferri et al., 2012). More specifically, it involves the set of institutions and processes for communicating, 
creating, delivering and exchanging products/services with value both for companies and consumers (D'Andrea et al., 2015a). Today an important role within the business domain is assigned to Social Media. Social Media allow companies collecting consumers' comments about products/services and performing better products/services development efforts as they can take into account consumers' needs (D'Andrea et al., 2011). Different studies have been focused on extracting consumer's sentiment on Social Media. An example is provided by Rastogi et al. (2014). The authors provided an approach for the extraction of consumer's sentiment of Facebook comments. The approach consists of the following steps: (i) data collection (ii) noise removal: (iii) classification: different domains such as: movies, technology products, economy, industry are used to classifies data (iv) name product/service recognition: a list of named entities is created that allow identifying important elements such as verb phrases, noun phrases, regular expressions (v) subjectivity classification: sentences are classified as objective/subjective (vi) features selection: the positive/negative sentiments are identified and combined (vii) sentiment extraction: different approaches are used such as supervised learning, unsupervised learning, and lexicon-based. Another example is given by Duwairi et al. (2014) with their approach for analysing Twitter comments. The approach is composed of the following phases: (i) collection dataset (ii) training dataset labeling by using crowd-sourcing (iii) analysis of the dataset to define the better methodology to use for normalizing the data (iv) normalization of the data in the preprocessing phase (v) creation of the feature vector for each entry in the dataset (vi) input of the feature vectors into the classifier for building the classification model (vii) use of the model to verify results. Another study is given by Khan et al. (2014) that provided an approach for performing the analysis and classification of Twitter comments. The approach involves two steps (i) processing that includes: removal of hash-tags URLs, username, and special characters etc. (ii) the hybrid scheme of classification algorithms for emoticon analysis and SentiWordNet analysis that improve the polarity classifier using a list of positive/negative words. A novel approach for the classification of online product reviews using sentiment analysis is provided by Bagwan et al. (2013). The approach uses appraisal words lexicon and product feature extraction for categorization of users reviews on the Amozon web site. The approach involves three main steps (i) dataset generation: the web crawling, a program for collecting the information from it is used (ii) feature extraction: it is done through the sentence segmentation, the tagging of speech part and the extraction of frequent features (iii) review categorization: each review is considered as a document for sentiments extraction. Finally, in De Clercq et al. (2014) an approach which finds disputed topics in news media is provided. The approach is entirely unsupervised, requiring neither an upfront definition of possible topics nor annotated training data. It comprises four steps (i) data collection (ii) sentiment analysis to determine the document's semantic orientation (iii) topic extraction to identify the topics of the news articles (iv) disputed categories extraction to identify disputed categories and hence topics. The analysis of these studies allows observing that only the sentiments extraction has been performed without considering the analysis of sentiment changes and the influence that these changes can have on the business strategy. User's opinions about a product/service may change from time to time, so the detection of these changes is important to reduce their impact to an acceptable level. In this context, the study provided by Bifet et al. (2011) provides an approach for analyzing, in real time, sentiments expressed in tweets. For the analysis, a system that uses an adaptive frequent item miner for data streams is provided. Another example is provided in the study conducted by Daas \& Puts (2014) that reveals changes in the sentiment of Dutch social media messages (Facebook and Twitter) and consumer confidence. Two different steps of the sentiment changes detection have been considered for the analysis: sentiment determination and data selection/analysis. Also in the study provided by Nguyen et al. (2012) a strategy to predict collective sentiment dynamics is provided. The collective sentiment changes are modeled by using a machine learning model without an analysis of individual tweets and their corresponding network structures. The following steps are considered for the analysis: (i) data collection (ii) feature extraction: tweets are analysed with respect to their complex level network structures (iii) sentiment analysis: the machine-learning-based and the Dynamic Language Model and lexicon-based Constrained Symmetric Nonnegative Matrix Factorization are used for the analysis (iv) sentiments' changes prediction: a simple approach to predicting the change of sentiment ratio using heuristics has been used. (v) identification of significant features: that contributes to the change of sentiment (vi) multi-class classification for predicting business strategy direction and ratio quantity. As for the studies that only consider the sentiment extraction, in these studies only the sentiment changes detection is discussed without both a detailed description of sentiment extraction and sentiment prediction phases. The sentiment prediction represents another important level of the sentiment analysis process even if, according to Sharma \& Lal (2012) "it is quite complex...many variables are involved and their tendency to interact with \& influence each other......". For this reason, the provided studies do not illustrate the steps to follow but only the use of different predictive models for the analysis of social/economic contexts that directly affect consumer attitudes and behaviours (Park, 2004; Madrigal, 2001;). 
Examples of prediction analysis are given in (Zhang et al., 2001a; Bollen et al., 2011; Gilbert \& Karahalios, 2010) focused on the economic domain. In particular, they underlined how consumer's mood, measured through social media, is related to stock market movements: demonstrating that anxiety and fear predate downward movements in the markets. In the following Table 1 the different studies are classified according to the different phases of the analysis.

Table 1. Classification of studies according to the different phases of the analysis

\begin{tabular}{lc}
\hline \multicolumn{1}{c}{ STUDIES } & PHASES OF SENTIMENT ANALYSIS \\
\hline Rastogi et al. (2014) & SENTIMENT EXTRACTION \\
Duwairi et al. (2014) & \\
Khan et al. (2014) & SENTIMENT CHANGES DETECTION \\
Bagwan et al. (2013) & \\
De Clercq et al. (2014) & \\
\hline Bifet et al. (2011) & \\
Daas \& Puts (2014) & \\
Nguyen et al. (2012) & \\
Madrigal (2001) & \\
\hline Park (2004) & \\
Madrigal (2001) & \\
Zhang et al. (2011a) & \\
Bollen et al. (2011) & \\
Gilbert \& Karahalios (2010) & \\
\hline The analysis of the literature allows individuating the different phases to consider for the creation of the \\
methodology. Each phase involves the different steps and some examples of approaches/methods/models to \\
perform them. In the following section, the methodology is described in detail.
\end{tabular}

\section{An Integrated Methodology for Sentiment Analysis in Business Domain}

This section describes the methodology (shown in Figure 1), which allows representing the entire process of sentiment analysis from sentiment extraction, through change detection to sentiment influence on the business strategy. The sentiment extraction phase allows analysing the user' opinion about a product/service in a past time period; it answers the following question: what happened? While the changes detection phase allows discovering the current user' opinion about product/services; it answers the following question: what is happening now? Finally, the sentiment prediction phase allows predicting how user' opinion influences the business strategy of the company; it answers the following question: what is going to happen in the future?

In the following sub-sections, these levels of the analysis are described in detail.

\subsection{First Phase: Sentiment Extraction}

The first phase allows detecting the user' sentiments on a particular product/service. It involves the: (i) Social Media monitoring (ii) features extraction (iii) data preparation (iv) sentiment classification. In the following sub-sections, these steps are described in detail.

\subsubsection{Social Media Monitoring}

Social Media monitoring allows extracting user' comments associated with specific product/service. There are many ways companies can approach Social media monitoring. The first approach is the simple observation. In many cases users are the best critics, so if a company has rolled out a new product/service and it wants to see what users think of it, their observation within the Social media environment can provide a big picture at a glance that allows knowing whether the general mood towards the product/service is positive or negative. Other companies that have more resources to dedicate to Social media monitoring can use a more proactive approach by engaging people who mention their brand in the social space. An example is FedEx that engaged users who mention them on Twitter. 


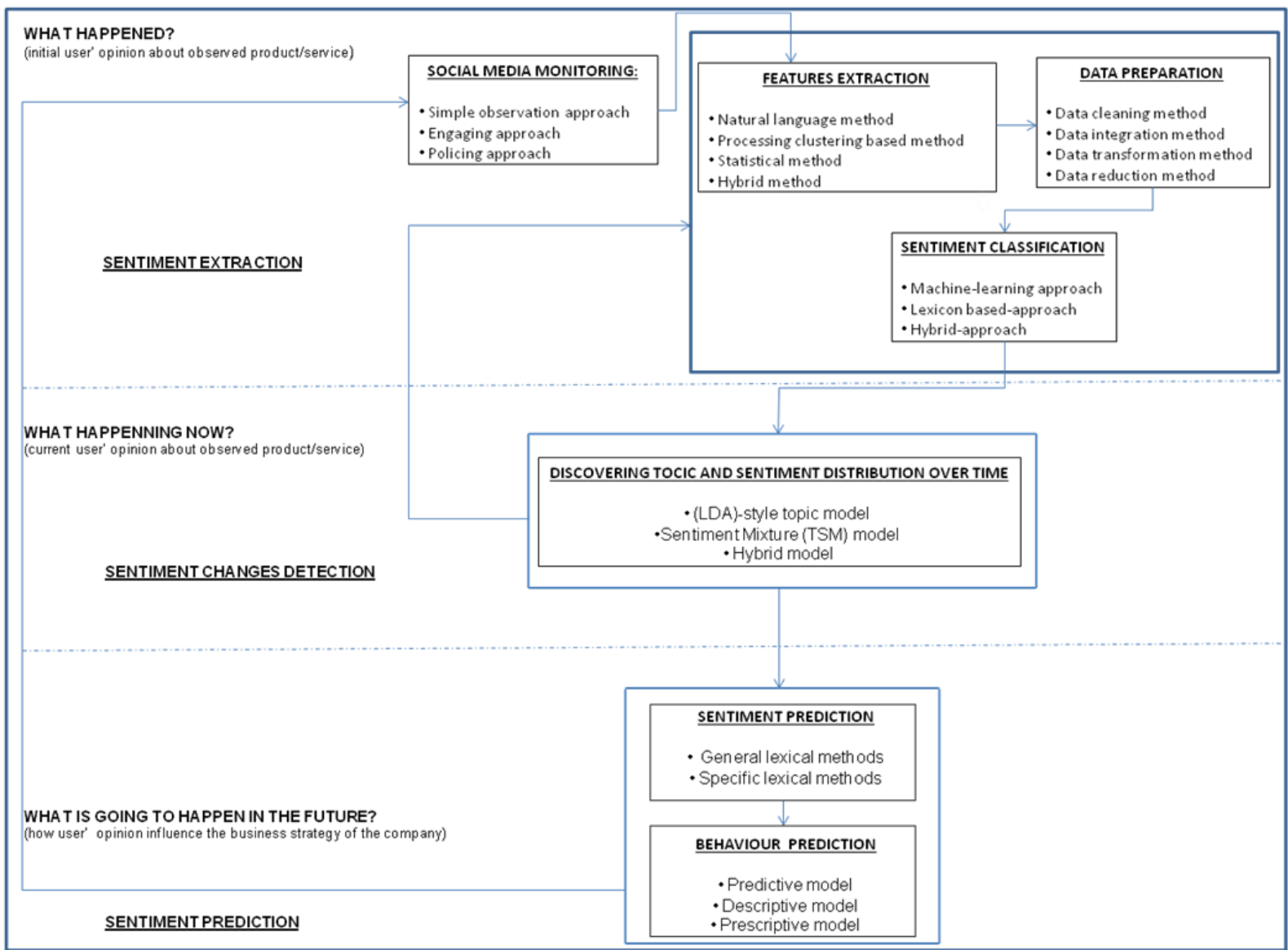

Figure 1. The structure of the methodology

\subsubsection{Features Extraction}

The second step consists in extracting the features that the company wants to consider for the sentiment analysis implementation. For the features extraction different methods can be used: natural language processing, clustering based, statistical and hybrid (Asghar et al., 2014). Natural language processing based methods mainly operate on: (i) noun-phrases, noun, adverbs, adjectives that usually express product features (Kobayashi et al., 2007; Archak et al., 2007), (ii) terms occurring near subjective expressions that can act as features (Kim \& Hovy, 2006). These methods have got high accuracy, but low recall with dependency on the accuracy of part of speech of tagging. Clustering based feature extraction methods (Zangh et al., 2011; Hadano et al., 2011; Zhang et al., 2012; Zhai et al., 2011) require few parameters to tune (Ganesan \& Zhai, 2012). The key weakness of these methods is that only major features can be extracted (Zhang et al., 2011b). While statistical methods are divided into three categories: univariate, multivariate and hybrid (Abbasi et al., 2011; Koncz \& Paralic, 2001). Examples of univariate methods include information gain occurrence frequency, log likely-hoodand minimum frequency thresholds. While decision tree models, recursive feature elimination and genetic algorithms represent examples of multivariate methods. As compared to univariate, multivariate methods are expensive in terms of computational efficiency, as they evaluate attribute interactions. A middle ground is represented by hybrid methods that combine univariate multivariate and other methods for achieving accuracy and efficiency (Balakrishnan et al., 2004; Liu \& Yu, 2005). Finally, the use of a hybrid method is provided in Hu \& Liu (2004) in which POS Tagging is combined with WordNet dictionary, while in Somprasertsri \& Lalitrojwong (2008) lexical and syntactic features are combined with a maximum entropy model.

\subsubsection{Data Preparation}

The data preparation step involves all the preparatory activities (pre-processing) for the analysis of user' comments such as removing non-textual contents and/or the markup tags and information that is not required for sentiment analysis. 


\subsubsection{Sentiment Classification}

Sentiment classification is a task of classifying a target unit in a document to positive or negative class (D'Andrea et al., 2015b). According to the way in which they are expressed in text, it can be also classified in explicit and implicit opinions. On considering the explicit opinions they are subjective statements that give regular/comparative opinions; while the implicit are objective statements that imply a regular or comparative opinion. Liu (2007) also gives a classification of opinions in: (i) direct opinions: directly expressed on a product/service or a product/service aspect (ii) indirect opinions: indirectly expressed on a product/service or a product/service aspect based on their effects. While Jindal \& Liu (2006) introduced comparative opinions that express a relation of similarities/differences between entities based on some aspects. The sentiment classification can be performed by using different approaches such as: (i) machine learning, (ii) lexicon-based and (iii) hybrid (Maynard \& Funk, 2011). Machine learning approach consists of two sets of documents: a training set and a test set. The training set is used for learning the differentiating characteristics of a document, while the test is used for checking how well the classifier performs. The main advantage of the machine learning approach is the ability to adapt and create trained models for specific purposes and contexts, while the limitation regards the general knowledge that may not be acquired from training data. The lexicon based approach uses sentiment dictionary with opinion words and matches them with the data for determining polarity. It has the advantage that general knowledge sentiment lexicons have wider term coverage, but it also presents two main limitations. Firstly, the number of words in the lexicons is finite, which may constitute a problem when extracting sentiment from very dynamic environments. Secondly, sentiment lexicons tend to assign a fixed sentiment orientation and score to words, irrespective of how these words are used in a text. Finally, in the hybrid approach, the combination of both the machine learning and lexicon based approaches has the potential to improve the sentiment classification performance. The main advantages of hybrid approaches are the lexicon/learning symbiosis, the detection and measurement of sentiment at the concept level and the lesser sensitivity to changes in the topic domain. While the main limitation is that reviews are with a lot of noise that assigns a neutral score because the method fails to detect any sentiment.

\subsection{Second Phase: Sentiments-Changes Detection}

Once user' sentiments have been extracted, another important step to consider is the sentiment changes detection. For detecting sentiments-changes it is important to: (i) discover topic distribution over time and (ii) identify sentiment changes. A useful way to analyse topic distribution over time is the time series that indicates the set of data points occurring at regular intervals. Whilst there are many complex mathematical time series techniques, it is also possible to focus on simple analyses of data extracted from Social media. These typically aggregate data into days to produce daily time series. According to Gruhl et al. (2004), three common patterns for topics can be extracted for the time series analysis:

- a single spike of interest that indicates a short period of time in which the product/service is discussed;

- fairly continuous discussion on the product/service without spikes;

- fairly continuous discussion on the product/service with occasional spikes triggered by relevant events.

As far as we know, there is no directly related work about sentiment change cause discovery so far. Some studies on topic's evolving trends over time provided methods such as the Latent Dirichlet Allocation (LDA)-style topic model provided by Wang \& McCallum (2006) that integrate the time factor into a probabilistic topic model and learn topic's distribution over time based on the topic model. Another example is the Topic-Sentiment Mixture model (Mei et al., 2007) that count the number of word occurrences of each topic to estimate it's dynamic over time. In Jiang et al. (2011) a hybrid solution to sentiment change detection is provided consisting of a combination of two different methods: probabilistic topic and language grammar that allows detecting steady topic sentiment changes in consecutive time periods. Once the topic distribution over time has been properly analysed; it is also important to identify the possible sentiment changes. Users can really modify their opinions about products and/or services according to the Social Influence process (Grifoni et al., 2013). The user opinion evolves as a result of interaction with other users that, in turn, e volve their opinion as a result of interaction with the complex network of social influences. This level of the analysis it is very important to adjust the business strategy in alignment with the user'opinion.

\subsection{Third Phase: Sentiment Prediction}

In order to have a global vision of the analysed product/service, it is important to perform the user' sentiment prediction. The goals of the sentiment prediction are (i) predicting the change of sentiment of a given topic over time (ii) identifying key features that contribute to the change. The majority of currently used methods for 
sentiment prediction are based on supervised learning techniques with the features derived from pre-existing sentiment lexical (Rosenthal et al., 2014; Rosenthal et al., 2015). Previous sentiment lexicons methods were developed as general-purpose resources (Liu \& Yu, 2005, Strapparava \& Valitutti, 2004; Wilson et al., 2005). Recently, an increasing amount of work specific lexicons methods has been developed (Mohammad, 2012). However, general and specific lexical methods still suffer from ambiguities at a contextual level. In order to overcome this limitation, Flekova et al. (2015) introduced a method for identifying frequent bigrams to assess the suitability of generic sentiment lexicons for a given domain. The provided method allows enhancing the assessment of lexicon based sentiment detection algorithms. The analysis of sentiment prediction is preliminary to understand how user' opinion can influence the business strategy. There are three types of models to perform behavior prediction: (i) predictive (ii) descriptive and (iii) prescriptive. The predictive model turns data into actionable, valuable, information. It uses data for determining probable future business outcomes. While the descriptive model looks at past business performance analyzing historical data to look for the reasons behind past success or failure. Finally, the prescriptive model automatically synthesizes big data, business rules for making predictions and suggesting decision options to take advantage of the predictions.

\section{Scenario: Analyse the User' Sentiment about WFG Financial Services}

The financial market analysis aims to analyse the general financial market for revealing discrepancies of financial services. Financial services reflect all available information, making it impossible to predict their changes based on past data (Bodie et al., 2011). Among this information, an important role is assumed by the user's sentiments that represent an important activity for determining financial products/services to offer (Zhu \& Niu, 2016). Starting from these considerations we illustrated how the methodology can be used for extracting user's sentiment on financial services. The analysis has been carried on Facebook where there are a lot of pages and/or group that allows members discussing financial issues. Among them, some examples are illustrated in Figure 2.

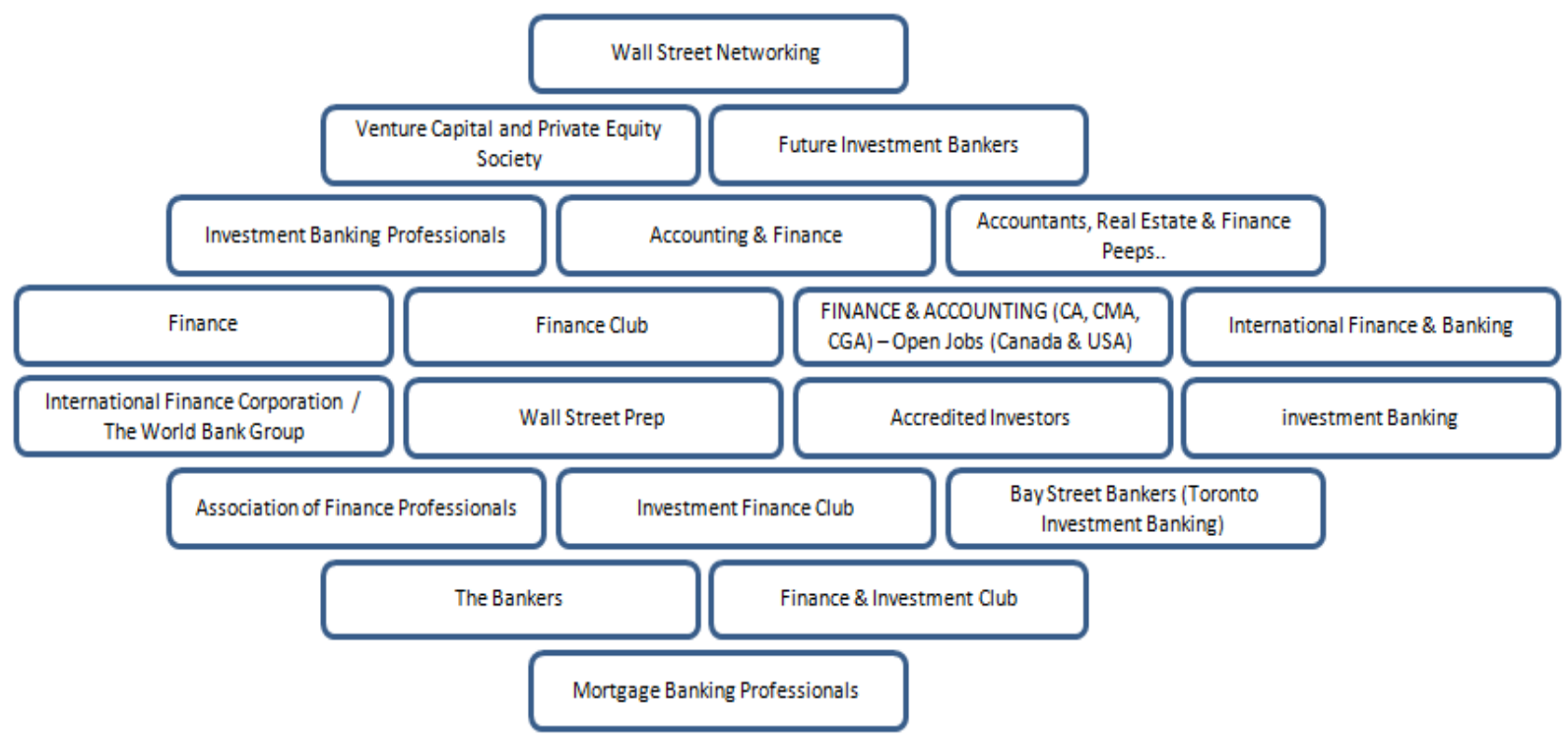

Figure 2. Examples of financial groups on Facebook

The methodology has been applied to extract users' opinion about financial services provided by the World Financial Group (WFG) company (Figure 3). 


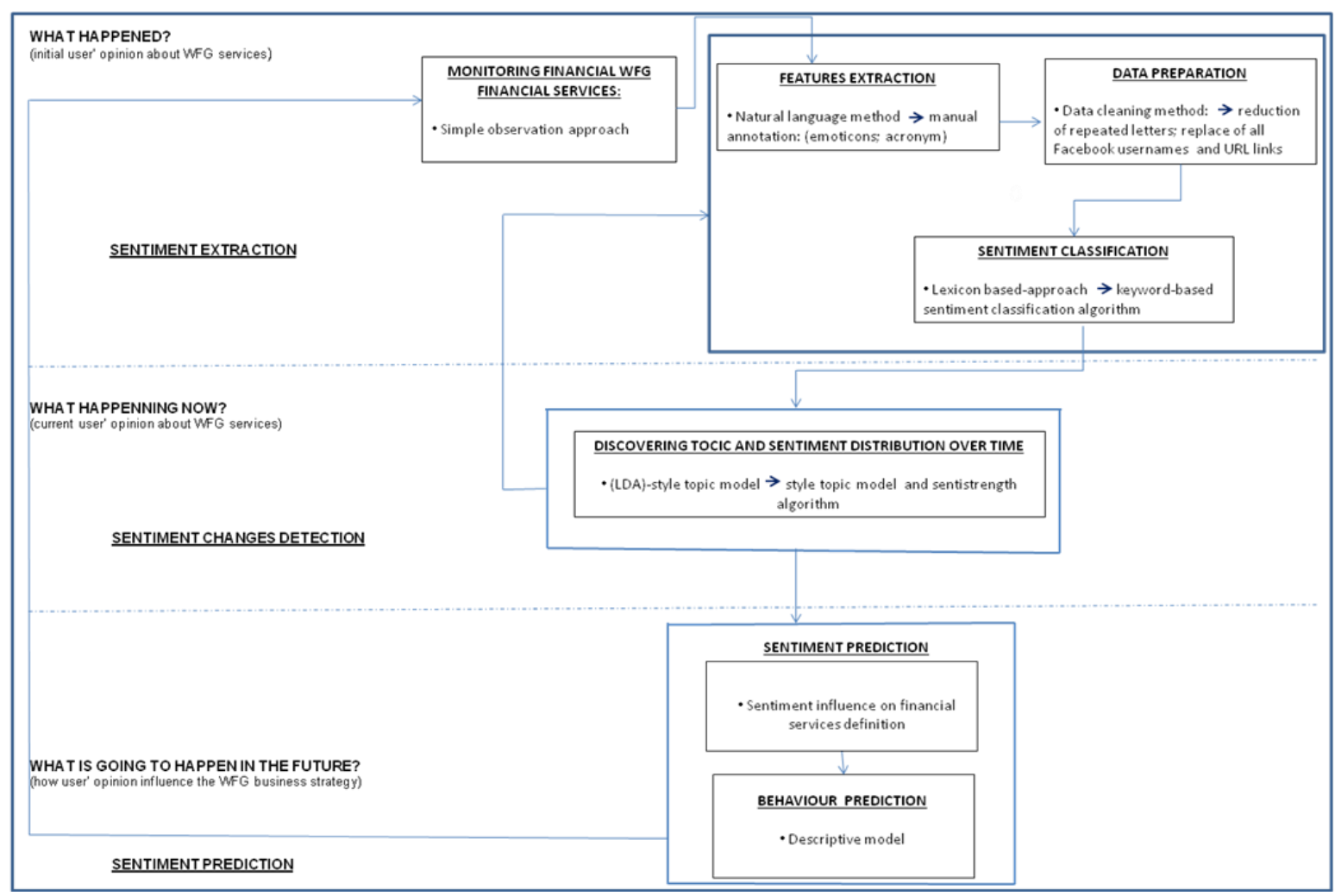

Figure 3. The methodology applied to the analysis of the WBF services/products

In the following sub-sections, the different steps of the analysis are described in detail.

\subsection{First Phase: Sentiment Extraction}

WFG is one of the few companies dedicated to serving the financial needs of individuals and families from all walks of life. It provides different kinds of services (as shown in Table 2):

Table 2. Financial services offered by the WFG

Life Insurance
Annuities
Critical Illness Insurance
Disability Insurance
Long-Term Care
Mutual Funds
Segregated Funds
Group Insurance
Business Solutions
College/Post-Secondary Education Funding
Investment Advisory Services
Financial Needs Analysis

\subsubsection{Social Media Monitoring}

The analysis has been carried out in two different steps. The first step refers to the first six months of the year 2016 (January 2016-June 2016) while the second step regards the last six month (July 2016-December 2016). For the analysis, two corpus data (for each period of the analysis) have been sampled and manually examined. The analysis has been restricted to the English language in order to remove the complication of multiple languages.

\subsubsection{Features Extraction}

The features extraction of the collected corpus data has been carried out at the sentence level. This level of the analysis consisted in extracting the different users' comments to each post published on the homepage of the group that we defined as "homepage posts". During the first period of the analysis 101 homepage posts have been published during the entire period of the analysis as shown in Figure 4. 


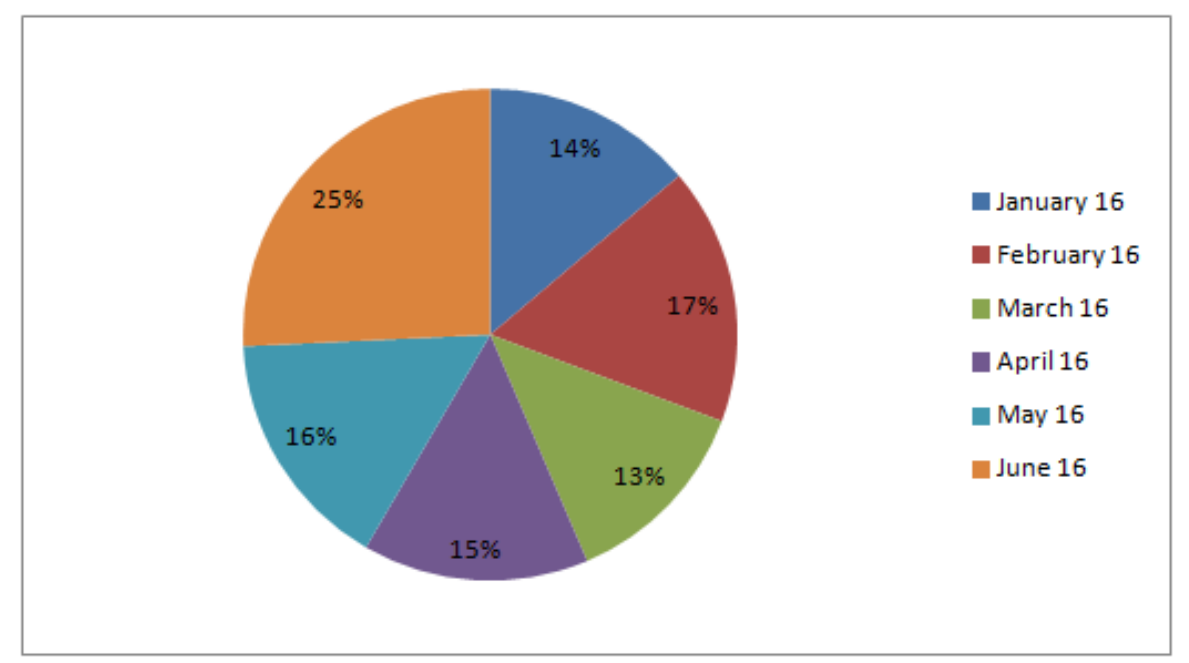

Figure 4. Homepage posts published during the first period of the analysis

As shown in the graphs the month that received the higher number of homepage posts was June followed by February and May. For each homepage posts the number of "like" and "sharing" is considered. In the following graphs (Figure 5) a representation of the number of "like" and "sharing" received by the homepage posts during the first six mounts of the analysis is given.

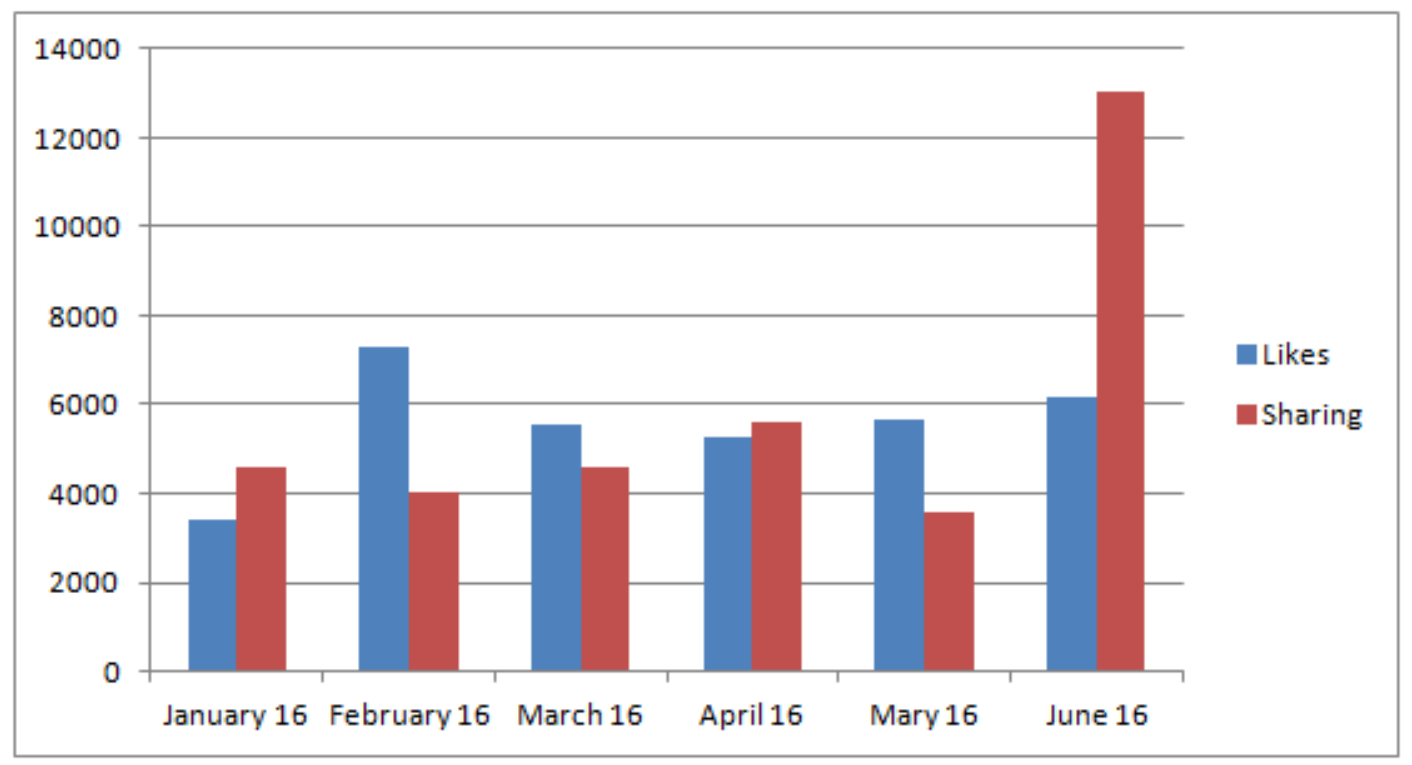

Figure 5. Number of "like" and "sharing" obtained by homepage posts during each month

The graph shown that no homepage posts have got at the same time the higher number of "likes" and "sharing". In fact the homepage posts that received the higher number of "likes" has been published in February while the homepage posts that received the higher number of "sharing" was published in June (even if it is important to highlight that the homepage posts published in September received the second higher number of "likes"). The different contents of the homepage posts have been analysed in order to classify them according to the different services provided by the WFG (summarised in Table 1). This classification shown that different financial services are discussed during the first six months of the analysis (Figure 6). 

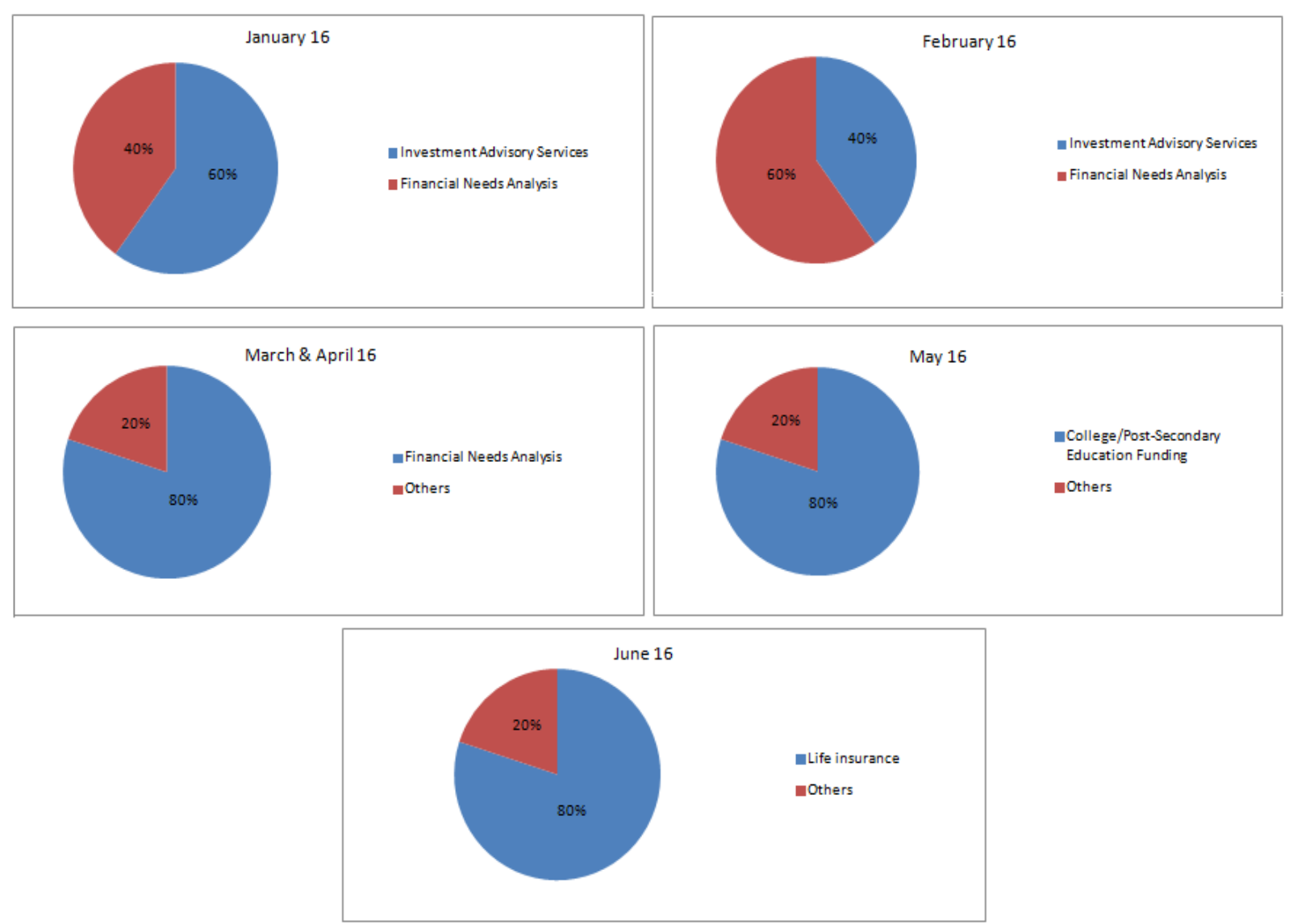

Figure 6. Contents of the homepage posts classified according to the WFG services

The most WFG services discussed in January was the "Investment advisory board" followed by the "Financial needs analysis" while the services most discussed in February was the "Financial needs analysis" followed by the "Investment advisory board". During March and April, discussion topics have been focused on "Financial need analysis" service while in May the most discussed service was the "College/Post secondary Education Funding". Finally, in June the most discussed service was the "Life Insurance".

For the analysis of user's sentiment on the different WFG services, the different comments, posted by people to the different homepage posts, have been considered (for the number of comments received by the homepage posts the first six months of the analysis see Table 3 ).

Table 3. Number of users' comments to homepage posts

\begin{tabular}{cc}
\hline Month & Total number of English comments \\
\hline January 16 & 71 \\
February 16 & 166 \\
March 16 & 71 \\
April 16 & 73 \\
May 16 & 43 \\
June 16 & 310 \\
\hline
\end{tabular}

The analysis allowed determining whether each comment expresses a positive, negative, or neutral opinion. For the features extraction, the natural language processing based method has been used. A random subset of 734 comments for the first six months of the analysis has been manually annotated. For the manual annotation of the users' comments three different elements have been considered:

- emoticons: facial expressions pictorially represented using letters and punctuation that express the user's mood. For the analysis, an emoticon dictionary by labeling 170 emoticons listed on Wikipedia with their emotional state can be used. For example, ":)" was labeled as positive whereas wile ":(" was labeled as negative. We assigned each emoticon a label from the following set of labels: Extremely-positive, Extremely-negative, Positive, Negative, and Neutral.

- acronym: an acronym dictionary from an on-line resource extract from http://www.dictionary.com/ has been used. 


\subsubsection{Data Preparation}

The collected comments were very noisy with a large number of irregular words; in order to reduce the noise the following pre-processing steps have been implemented:

- reduction of letters that are repeated more than twice in all words. For example, the word "insuranceeeeeeeee" becomes "insurance" after reduction.

- replace of all Facebook usernames, with the term "USER".

- replace of all URL links in the corpus with the term "URL".

\subsubsection{Sentiment Classification}

To perform the sentiment classification two discriminatory word lexicons, one containing words indicating positive sentiment and another one containing words indicating negative sentiment were built. If a comment had an equal number of positive and negative keywords, it was a tie while if it contained neither negative nor positive keywords, it was neutral. The analysis showed that the corpus data got mostly positive comments. It is important to note here that annotating comments was not easy. Many times, it was difficult to determine the sentiment. Some comments appeared both negative and positive; this is because neutral comments had both negative and positive sentiments.

\subsection{Second Phase: Sentiments-Changes Detection}

During the second period of the analysis (July 2016-December 2016) 134 homepage posts have been published distributed as shown in Figure 7.

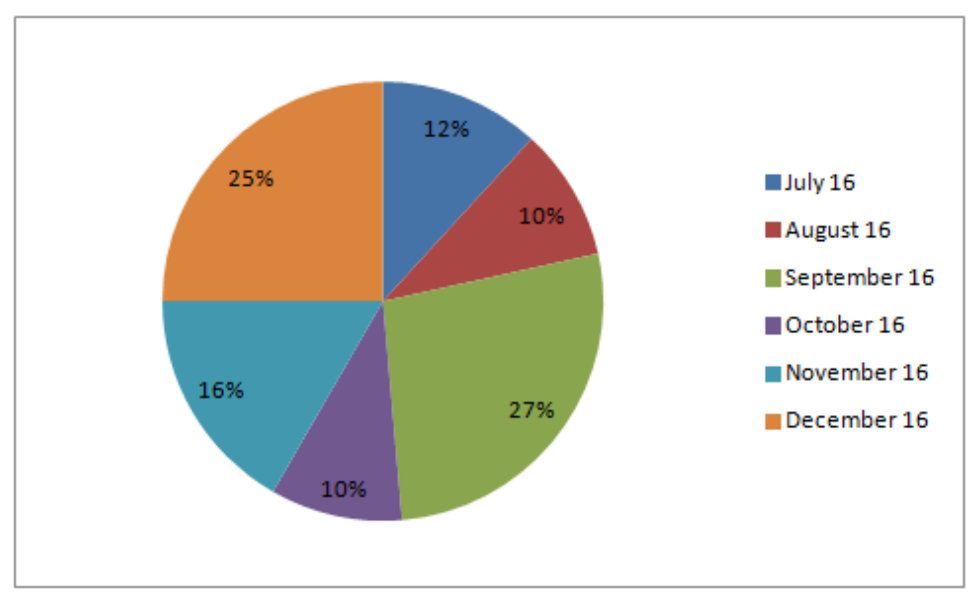

Figure 7. Homepage posts published during the second period of the analysis

As shown in Figure 8 the month that received the higher number of homepage posts is September followed by December.

For each homepage posts the number of "like" and "sharing" is considered. In the following graph (Figure 8) a representation of the number of "like" and "sharing" obtained by homepage posts during the second period of the analysis is given. 


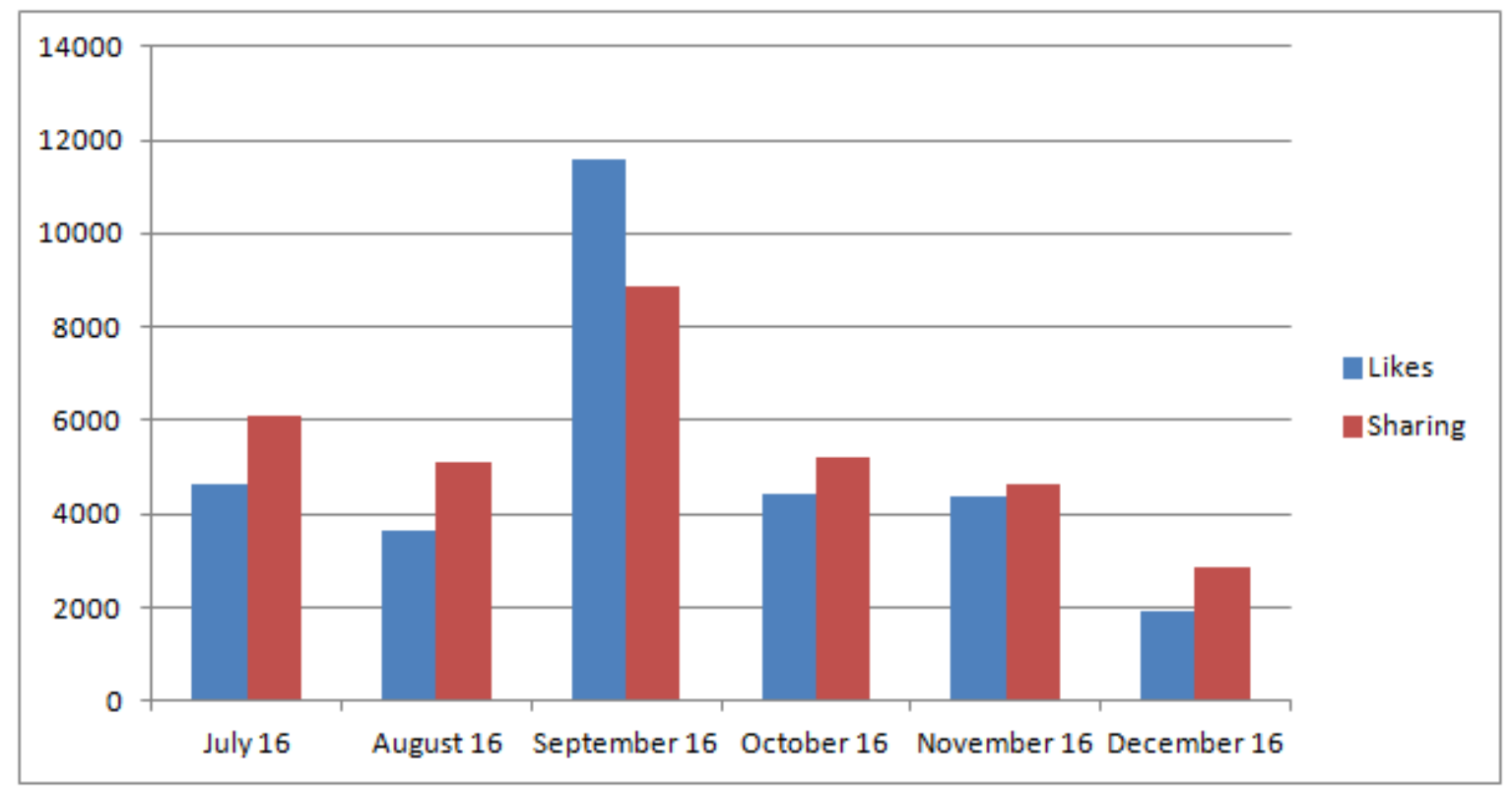

Figure 8. Number of "like" and "sharing" obtained by homepage posts during each month

The graph shown that the homepage published in September received at the same time the higher number of "likes" and "sharing". As for the first period of the analysis, the contents of the different homepage posts have been classified according to the different WFG services (Figure 9). Note that the "others" class refers to homepage posts that it was not possible to classify in the different WFG services (an example is represented by the announcement of the WFG annual convention that received a higher number of "like" and "sharing" emphasizing the positive vision of users about the WCG services).
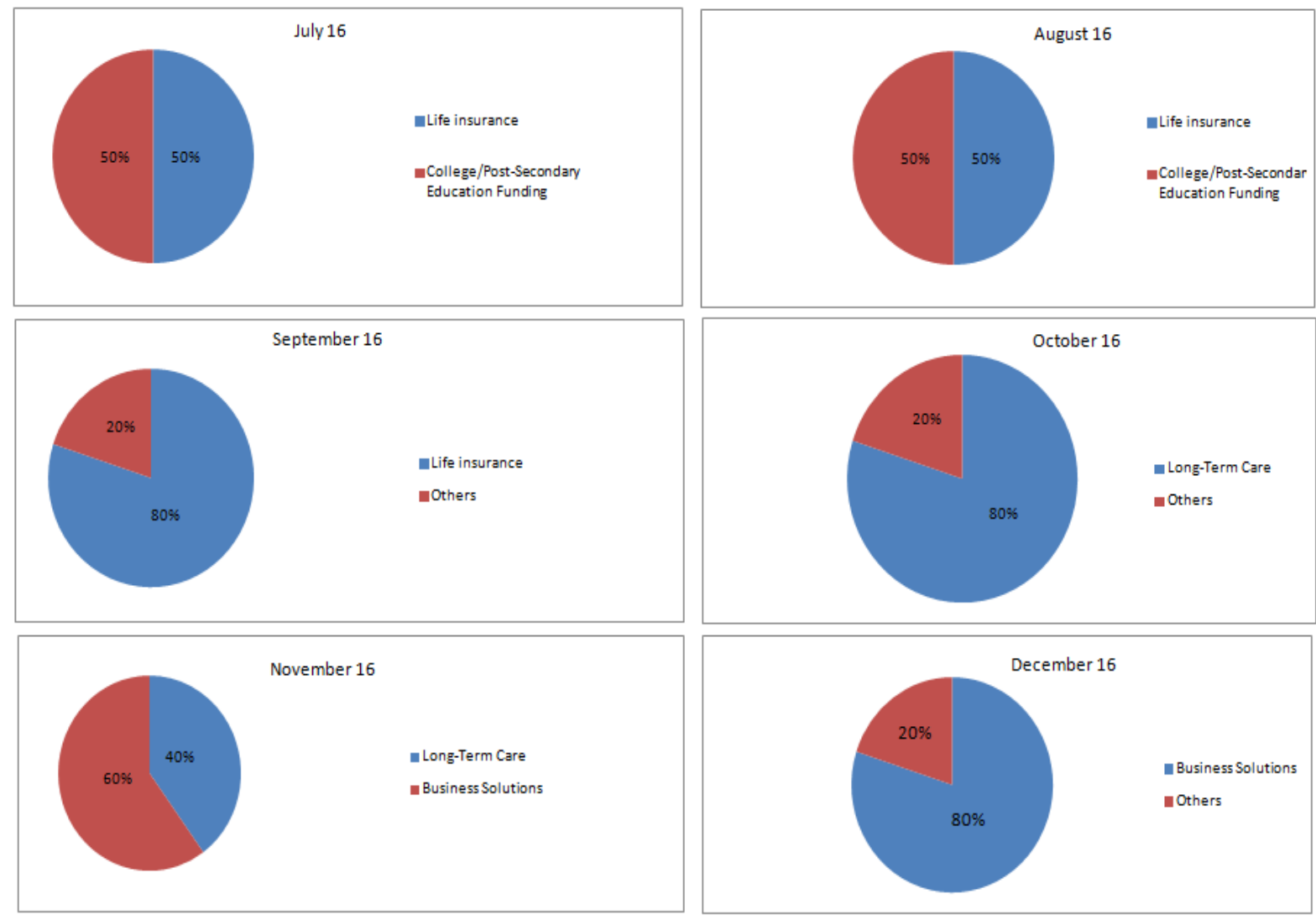

Figure 9. Contents of the homepage posts classified according to the WFG services 
The WFG services most discussed in July were the "Life Insurance" and the "College/Post Secondary Education Founding". The "Life insurance" service continued to be discussed also in August together with the "College/Post-Secondary Education and in September while in October the "Long-Term Care" service was the most discussed. The Long-Term Care" service continued to be discussed also in November together with the "Business solutions" service. Finally, in December the most discussed service was the "Business solution". Also in this second step for the analysis of users sentiment on the different WFG services the different comments, posted by people to the different homepage posts, have been considered (for the number of comments received by the homepage posts the second six months of the analysis see Table 4). Arandom subset of 342 comments for the second six months of the analysis has been manually annotated.

Table 4. Number of users' comments to homepage posts

\begin{tabular}{cc}
\hline MONTH & $\begin{array}{c}\text { TOTAL NUMBER OF ENGLISH } \\
\text { COMMENTS }\end{array}$ \\
\hline July 16 16 & 69 \\
August 16 & 44 \\
September 16 & 51 \\
October 16 & 110 \\
November 16 & 42 \\
December 16 & 26 \\
\hline
\end{tabular}

After all opinions have been properly extracted, it was important to analyse how to obtain the properly aggregated sentiment distribution over time. The top comment from the selected days have been identified using the time series scanning method (Thel wall et al., 2006; Thelwall \& Prabowo, 2007) that separately calculates for each word in the entire corpus its hourly relative frequency (proportion of comments per hour containing the word) and then the largest increase in relative frequency during the time period. This method thus created a list of services with the biggest spikes of interest. The top 20 comments identified through the above method were not all chosen as the main service because in some cases multiple words referred to the same service. The next stage was to classify the sentiment strength of each comment. Whilst many algorithms detect text subjectivity or sentiment polarity, a few detect sentiment strength (Wilson et al., 2006; Strapparava \& Mihalces, 2008). Nevertheless, the accurate detection of sentiment is domain-dependant. The SentiStrength (http://sentistrength.wlv.ac.uk/ a) seemed to be more appropriate than the most similar published algorithm (Neviarousskaya et al., 2007) because the latter had fewer features and has been less extensively tested for accuracy. SentiStrength classified for positive and negative sentiment on a scale of 1 (no sentiment) to 5 (very strong positive/negative sentiment). All comments have been classified for positive and negative sentiment strength and then hourly average positive and negative sentiment strength scores were calculated for each service. The analysis showed that also in this second period of the analysis the corpus data got mostly positive comments.

\subsection{Third Phase: Sentiment Prediction}

Based on our observation on the two testing corpuses, we found if the user opinion about the financial services offered by the WFG changed. We repeated this monitoring step during a long period of time after the end of the analysis to have a concrete vision of the effective sentiment change. The analysis of the sentiment change (in a positive or negative way) also considers the number of "likes" and "sharing" obtained by the different homepages posts. This analysis highlighted that not significant changes occurred; all services have been evaluated in a positive way by the users also during the second phase of the analysis. However, it is possible to highlight some differences. On considering the first period of the analysis all services are considered by users in a positive way with some differences. The service estimated in a more positive way was the "Life insurance" and this has been also confirmed by the obtained number of "sharing" of this service during the entire period of the analysis. The second service in the users' ranking was the "College/Post secondary Education Funding" service, followed by "Investment Advisory board" and "Financial need analysis" services. Also in the second phase of the analysis, all services are considered by users in a positive way with some differences. The service estimated in a more positive way was the "Long term Care" and this has been also confirmed by the number of "likes" and "sharing" obtained by this service during the entire period of the analysis. In the second position there was the "Life insurance" and also in this case this result has been confirmed by the number of slikes" obtained by this service during the entire period of the analysis. The service that, during the entire period of the analysis, obtained the higher number of positive comments (summed to the number of "like" and "sharing") was "Life insurance" followed by the "Long term Care" and the "College/Post secondary Education Funding). These results underlined the important role of insurance services for users. Insurance services support users in keeping his/her financial situation stable; they decrease the level of unnecessary (individual) precautionary savings and enable capital to be allocated to higher-return projects. Thus, insurance stimulates investment and consumption by 
reducing the amount of capital bound in relatively unproductive areas. This aspect can represent an important element for the WFB companies for understanding and supporting decision-making for financial services as well as for creating more appropriate business strategies which may help users make improved decisions.

\section{Discussion}

Focusing on the importance of sentiment analysis processing within the business strategies this paper provided the methodology. The choice to develop methodology was made to achieve two different aims:

- to provide guidelines to companies that want to develop sentiment analysis within their business strategies;

- to overcome the fragmentation of the existing literature in this field.

Containing a description of the different phases and steps that compose the sentiment analysis process and providing examples of the different approaches to use in each of them, the methodology allows companies simplifying the implementation of sentiment analysis process and investing their resources (time, budget and staff) - previously dedicated to the individuation of the different components of the sentiment analysis process to other business objectives. For the development of the methodology we start from the assumption that to develop an efficient sentiment analysis in business domain it is important not only to consider the extraction of sentiments on identified products/services but also the sentiments changes over time, underlining the possible causes of these changes, and the sentiment prediction for the implementation of future business strategies. The existing literature is very fragmented in this context; in fact, in some studies, only a description of the sentiment extraction phase is provided while other studies only consider sentiment changes and/or sentiment prediction phases. The provided methodology allows overcoming this fragmentation; the advantages of are double:

- it allows implementing the entire process of sentiment analysis simultaneously

- it allows focusing on the different phases of the sentiment analysis process in different periods of time in an integrated manner without missing data collected during the different phases.

\section{Conclusion and Future Works}

The paper provided a methodology for approaching sentiment analysis in business domain composed of different phases that compose the sentiment analysis processing. An example of an application scenario has been defined illustrating how the methodology can be used for extracting user' sentiment about financial services of the WFG company. The case study represented a first application of the methodology for exploiting the potential. Future works will dress the external and internal validation of the provided methodology. The external validation will consist on the implementation of different case studies on other Social Media such as Twitter with different level of complexity. While the internal validation will consist on reflections that will aim to assess the characteris tics of the methodology based on the set of future steps that able to improve it.

\section{References}

Abbasi, A., France, S., Zhang, Z., \& Chen, H. (2011). Selecting attributes for sentiment classification using feature relation networks. IEEE Transactions on Knowledge and Data Engineering, 23(3), 447-462. https://doi.org/10.1109/TKDE.2010.110

Archak, N., Ghose, A., \& Ipeirotis, P. G. (2007, August). Show me the money!: deriving the pricing power of product features by mining consumer reviews. In Proceedings of the 13th ACM SIGKDD international conference on Knowledge discovery and data mining, 56-65. ACM.

Asghar, M. Z., Khan, A., Ahmad, S., \& Kundi, F. M. (2014). A Review of Feature Extraction in Sentiment Analysis. Journal of Basic and Applied Scientific Research, 4(3), 181-186.

Bagwan, A. B., Chaudhari, D., \& Deshmukh, R. A. (2013). A framework for Review Categorization using Appraisal words. International Journal of Advanced Research in Computer and Communication Engineering, 2(4).

Balakrishnan, P. V., Gupta, R., \& Jacob, V. S. (2004). Development of hybrid genetic algorithms for product line designs. IEEE Transactions on Systems, Man, and Cybernetics, Part B (Cybernetics), 34(1), 468-483. https://doi.org/10.1109/TSMCB.2003.817051

Bifet, A., Holmes, G., Pfahringer, B., \& Gavalda, R. (2011, October). Detecting sentiment change in Twitter streaming data. In Proceedings of the Second Workshop on Applications of Pattern Analysis, 5-11.

Bodie, Z., Kane, A., \& Marcus, A. J. (2011). Student solutions manual for" Investments". McGraw-Hill Irwin. 
Bollen, J., Mao, H., \& Zeng, X. (2011). Twitter mood predicts the stock market. Journal of Computational Science, 2(1), 1-8. https://doi.org/10.1016/j.jocs.2010.12.007

Daas, P. J. H., \& Puts, M. J. H. (2014). Social media sentiment and consumer confidence. Workshop on using Big Data for Forecasting and Statistics, Frankfurt, Germany.

D'Andrea, A., Ferri, F., \& Grifoni, P. (2011). A Business Model Framework for Second Life. Book "E-Novation for Competitive Advantage in Collaborative Globalization: Technologies for Emerging E-Business Strategies". Edited by: Hugh M. Pattinson \& David R. Low. IGI Publishing, 2011.

D'Andrea, A., Ferri, F., \& Grifoni, P. (2012). SNeM2S: a Social Network Model for Marketing Strategies. International Journal of e-business development; The World Academic Publishing Co, Limited, Hong Kong (Cina)

D'Andrea, A., Ferri, F., \& Grifoni, P. (2015a). CBM: An Integrated Crowd-sourcing Business Model. Journal of Contemporary Management, 5(2), 47-58

D'Andrea, A., Ferri, F., Grifoni, P., \& Guzzo, T. (2015b): Approaches, Tools and Applications for Sentiment Analysis Implementation. International Journal of Computer Applications, 125(3). https://doi.org/10.5120/ijca2015905866

De Clercq, O., Hertling, S., Hoste, V., Ponzetto, S. P., \& Paulheim, H. (2014). Identifying Disputed Topics in the News. Linked Data for Knowledge Discovery (LD4KD), 37-48.

Duwairi, R. M., Marji, R., Sha'ban, N., \& Rushaidat, S. (2014). Sentiment Analysis in Arabic tweets. Information and Communication Systems (ICICS), 5th International Conference on IEEE, 1-6. https://doi.org/10.1109/IACS.2014.6841964

Fan, T. K., \& Chang, C. H. (2011). Blogger-centric contextual advertising. Expert Systems with Applications, 38(3), 1777-1788. https://doi.org/10.1016/j.eswa.2010.07.105

Ferri, F., D'Andrea, A., \& Grifoni, P. (2012). IBF: An Integrated Business Framework for Virtual Communities. Journal of electronic commerce in organizations. IGI Global, Hershey (Stati Uniti d'America). https://doi.org/10.4018/jeco.2012010101

Flekova, L., Ruppert, E., \& Preotiuc-Pietro, D. (2015). Analysing domain suitability of a sentiment lexicon by identifying distributionally bipolar words. 6th workshop on computational approaches to subjectivity, sentiment and social media analysis wassa. https://doi.org/10.18653/v1/W15-2911

Ganesan, K., \& Zhai, C. (2012). Opinion-based entity ranking. Information retrieval,15(2), 116-150. https://doi.org/10.1007/s10791-011-9174-8

Gilbert, E., \& Karahalios, K. (2010). Widespread worry and the stock market. Proceedings of the Fourth International AAAI Conference on Weblogs and Social Media.

Grifoni, P., Ferri, F., \& D'Andrea, A. (2013). An integrated framework for on-line viral marketing campaign planning. International journal of business research, 6(1), 22-30. https://dx.doi.org/10.5539/ibr.v6n1p22

Gruhl, D., Chavet, L., Gibson, D., Meyer, J., \& Pattanayak, P. (2004). How to build a WebFountain: An architecture for very large-scale text analytics. IBM Systems Journal, 43(1), 64-77. https://doi.org/10.1147/sj.431.0064

Hadano, M., Shimada, K., \& Endo, T. (2011). Aspect identification of sentiment sentences using a clustering algorithm. Procedia-Social and Behavioral Sciences, 27, 22-31. https://doi.org/10.1016/j.sbspro.2011.10.579

Hu, M., \& Liu, B. (2004,). Mining opinion features in customer reviews. AAAI 4(4), 755-760.

Jiang, Y., Meng, W., \& Yu, C. (2011). Topic sentiment change analysis. In Machine Learning and Data Mining. Pattern Recognition 443-457. Springer Berlin Heidelberg. https://doi.org/10.1007/978-3-642-23199-5_33

Jindal, N., \& Bing, L. (2006). Mining comparative sentences and relations. Proceedings of National Conf. On Artificial Intelligence (AAAI-2006).

Khan, F. H., Bashir, S., \& Qamar, U. (2014). TOM: Twitter opinion mining framework using hybrid classification scheme. Decision Support Systems, 57, 245-257. https://doi.org/10.1016/j.dss.2013.09.004

Kim, S. M., \& Hovy, E. (2006). Automatic identification of pro and con reasons in online reviews. Proceedings of the COLING/ACL on Main conference poster sessions, 483-490. Association for Computational 
Linguistics. https://doi.org/10.3115/1273073.1273136

Kobayashi, N., Inui, K., \& Matsumoto, Y. (2007). Extracting Aspect-Evaluation and Aspect-Of Relations in Opinion Mining. EMNLP-CoNLL 7, 1065-1074.

Koncz, P., \& Paralic, J. (2011). An approach to feature selection for sentiment analysis. In Intelligent Engineering Systems (INES), 2011 15th IEEE International Conference, 357-362. https://doi.org/10.1109/INES.2011.5954773

Liu, B. (2007). Web data mining: exploring hyperlinks, contents, and usage data. Springer Science \& Business Media.

Liu, H., \& Yu, L. (2005). Toward integrating feature selection algorithms for classification and clustering. Knowledge and Data Engineering, IEEE Transactions, 17(4), 491-502. https://doi.org/10.1109/TKDE.2005.66

Madrigal, R. (2001). Social Identity Effects in a Belief-Attitude-Intentions Hierarchy: Implications for Corporate Sponsorship. Psychology and Marketing, 18(2), 145-165. https://doi.org/10.1002/1520-6793(200102)18:2<145::AID-MAR1003>3.0.CO;2-T

Maynard, D., \& Funk, A. (2011). Automatic detection of political opinions in tweets. Proceedings of the 8th international conference on the semantic web, ESWC'11, 88-99. https://doi.org/10.1007/978-3-642-25953-1_8

Mei, Q., Ling, X., Wondra, M., Su, H., \& Zhai, C. (2007, May). Topic sentiment mixture: modeling facets and opinions in weblogs. In Proceedings of the 16th international conference on World Wide Web (pp. 171-180). ACM. https://doi.org/10.1145/1242572.1242596

Mohammad, S. M. (2012, June). \# Emotional tweets. In Proceedings of the First Joint Conference on Lexical and Computational Semantics-Volume 1: Proceedings of the main conference and the shared task, and Volume 2: Proceedings of the Sixth International Workshop on Semantic Evaluation (pp. 246-255). Association for Computational Linguistics.

Nguyen, L. T., Wu, P., Chan, W., Peng, W., \& Zhang, Y. (2012, August). Predicting collective sentiment dynamics from time-series social media. In Proceedings of the first international workshop on issues of sentiment discovery and opinion mining (p. 6). ACM. https://doi.org/10.1145/2346676.2346682

Park, C. (2004). Efficient or enjoyable? Consumers Vales of Eating-out and Fast Food Restaurant Consumption in Korea. International Journal of Hospitality Management, 23, 87-94. https://doi.org/10.1016/j.ijhm.2003.08.001

Rastogi, S. S. K.., Singhal, R., \& Kumar, R. (2014). A Sentiment Analysis based Approach to Facebook User Recommendation. International Journal of Computer Applications, 90(16), 21-25. https://doi.org/10.5120/15805-4670

Rosenthal, S., Nakov, P., Kiritchenko, S., Mohammad, S., Ritter, A., \& Stoyanov, V. (2015, June). SemEval-2015 Task 10: Sentiment Analysis in Twitter. In SemEval@ NAACL-HLT, 451-463. https://doi.org/10.18653/v1/S15-2078

Rosenthal, S., Ritter, A., Nakov, P., \& Stoyanov, V. (2014). Semeval-2014 task 9: Sentiment analysis in twitter. Proceedings of the 8th International Workshop on Semantic Evaluation (SemEval 2014), 73-80. https://doi.org/10.3115/v1/S14-2009

Sharma, S., \& Lal, K. (2012). Changing consumer behaviour-A challenge for sustainable business growth. International Journal of Marketing, Financial Services \& Management Research, 1(8), 149-158.

Somprasertsri, G., \& Lalitrojwong, P. (2008, July). Automatic product feature extraction from online product reviews using maximum entropy with lexical and syntactic features. In Information Reuse and Integration, 2008. IRI 2008. IEEE International Conference on (pp. 250-255). IEEE. https://doi.org/10.1109/IRI.2008.4583038

Strapparava, C., \& Mihalcea, R. (2008, March). Learning to identify emotions in text. In Proceedings of the 2008 ACM symposium on Applied computing (pp. 1556-1560). ACM. https://doi.org/10.1145/1363686.1364052

Strapparava, C., \& Valitutti, A. (2004). WordNet Affect: an Affective Extension of WordNet. In LREC, 4, 1083-1086. 
Thelwall, M., \& Prabowo, R. (2007). Identifying and characterising public science-related concerns from RSS feeds. Journal of the American Society for Information Science \& Technology, 58(3), 379-390. https://doi.org/10.1002/asi.20504

Thelwall, M., Prabowo, R., \& Fairclough, R. (2006). Are raw RSS feeds suitable for broad issue scanning? A science concern case study. Journal of the American Society for Information Science and Technology, 57(12), 1644-1654. https://doi.org/10.1002/asi.20334

Wang, C. J., Tsai, M. F., Liu, T., \& Chang, C. T. (2013). Financial Sentiment Analysis for Risk Prediction. Proceedings of the Sixth International Joint Conference on Natural Language Processing, 802-808

Wang, X., \& McCallum, A. (2006, August). Topics over time: a non-Markov continuous-time model of topical trends. In Proceedings of the 12th ACM SIGKDD international conference on Knowledge discovery and data mining (pp. 424-433). ACM. https://doi.org/10.1145/1150402.1150450

Wilson, T., Wiebe, J., \& Hoffmann, P. (2005, October). Recognizing contextual polarity in phrase-level sentiment analysis. In Proceedings of the conference on human language technology and empirical methods in natural language processing ( $\mathrm{pp}$. 347-354). Association for Computational Linguistics. https://doi.org/10.3115/1220575.1220619

Wilson, T., Wiebe, J., \& Hwa, R. (2006). Recognizing strong and weak opinion clauses. Computational Intelligence, 22(2), 73-99. https://doi.org/10.1111/j.1467-8640.2006.00275.x

Zhai, Z., Xu, H., Kang, B., \& Jia, P. (2011). Exploiting effective features for Chinese sentiment classification. Expert Systems with Applications, 38(8), 9139-9146. https://doi.org/10.1016/j.eswa.2011.01.047

Zhang, H., Yu, Z., Xu, M., \& Shi, Y. (2011, March). Feature-level sentiment analysis for Chinese product reviews. In Computer Research and Development (ICCRD), 2011 3rd International Conference on (Vol. 2, pp. 135-140). IEEE.

Zhang, S., Jia, W., Xia, Y., Meng, Y., \& Yu, H. (2011). Product features extraction and categorization in Chinese reviews. In The Sixth International Multi-Conference on Computing in the Global Information Technology, ICCGI.

Zhang, W., Xu, H., \& Wan, W. (2012). Weakness Finder: Find product weakness from Chinese reviews by using aspects based sentiment analysis. Expert Systems with Applications,39(11), 10283-10291. https://doi.org/10.1016/j.eswa.2012.02.166

Zhu, B., \& Niu, F. (2016). Investor sentiment, accounting information and stock price: Evidence from China. Pacific-Basin Finance Journal, 38, 125-134. https://doi.org/10.1016/j.pacfin.2016.03.010

\section{Copyrights}

Copyright for this article is retained by the author(s), with first publication rights granted to the journal.

This is an open-access article distributed under the terms and conditions of the Creative Commons Attribution license (http://creativecommons.org/licenses/by/4.0/). 